\title{
Isoleucine starvation caused by sulfometuron methyl in Salmonella typhimurium measured by translational frameshifting
}

\author{
Alexander Kaplun, David M. Chipman and Ze'ev Barak
}

Author for correspondence: Ze'ev Barak. Tel: +972 8 6461713. Fax: +97286461710. e-mail: barakz@bgumail.bgu.ac.il

Department of Life Sciences, Ben-Gurion University of the Negev, Beer-Sheva, 84105, Israel

\begin{abstract}
The authors have developed a tool for the study of inhibitor-induced amino acid starvation in bacteria which exploits the phenomenon of translational frameshifting. The inhibition of acetohydroxyacid synthase II by the herbicide sulfometuron methyl (SMM) has complex effects on branched-chain amino acid biosynthesis. Experiments were done with Salmonella typhimurium containing a plasmid with an isoleucine codon in a 'shifty' region, prone to translational frameshifting. SMM did not cause translational frameshifting in minimal medium under conditions that inhibit growth. A 20-fold higher concentration of SMM was required to cause starvation for isoleucine, e.g. in the presence of valine. This starvation was reflected in translational frameshifting correlated with inhibition of growth. These observations support the authors' previous suggestions based on other techniques. The method used here could be generalized for the study of complex metabolic effects related to amino acids.
\end{abstract}

Keywords: acetohydroxyacid synthase, acetolactate synthase, branched-chain amino acids, sulfonylurea, amino acid biosynthesis

\section{INTRODUCTION}

Sulfometuron methyl (SMM) and other sulfonylurea herbicides are potent and specific inhibitors of acetohydroxyacid synthase (AHAS), the first common enzyme in the biosynthetic pathway to the branched-chain amino acids (LaRossa \& Schloss, 1984; Schloss, 1984; Chaleff \& Mauvais, 1984); see Fig. 1. This enzyme catalyses the decarboxylation of pyruvate and its condensation with a second molecule of pyruvate to yield acetolactate, the precursor of valine (and leucine), or with 2-oxobutyrate to yield acetohydroxybutyrate, the precursor of isoleucine. Because of the branching of the pathway, feedback inhibition of several enzymes by end products, and competition between substrates of the same and different enzymes, the metabolic and physiological consequences of inhibition by sulfonylureas are not simple and are still not completely understood (Epelbaum et al., 1996, 1998; LaRossa et al., 1987; Jia et al., 2000). An understanding of the ramifications of AHAS inhibitors is important for the understanding of their mode of action as herbicides and for the development of new antimicrobial and herbicidal agents. Such investigations have been hampered by the lack of a

Abbreviations: AHAS, acetohydroxyacid synthase; ILHX, isoleucine hydroxamate; SMM, sulfometuron methyl. reliable method for direct and quantitative assessment of the degree of starvation for individual amino acids caused by the presence of the inhibitor. We report here the development of a tool for the quantitative study of inhibitor-induced amino acid starvation which exploits the phenomenon of translational frameshifting.

Translational frameshifting occurs when a ribosome under specific conditions shifts from the original reading frame $(0)$ in one $(+)$ or another $(-)$ direction. Such displacement, as a rule, is by one or two nucleotides (Atkins et al., 1990; Parker, 1989). As a result, the ribosomes produce two polypeptides with the same amino-terminal region, but differing in their carboxyterminal sequence, from the same mRNA. The probability of spontaneous translational frameshifting at a particular codon is very low, about $10^{-4}$, but the presence of certain 'shifty' sequences significantly increases it, sometimes up to more than $50 \%$ (Atkins et al., 1990). This special behaviour is, of course, naturally limited to specific genes, but is widespread among viruses (especially retroviruses) and is found in bacteria and even in humans (Ivanov et al., 2000).

To study the translational frameshifting mechanism, Gallant \& Lindsley (1992) developed a bacterial system in which an original 'shifty' region to be studied is inserted after the lac promoter and the translational 


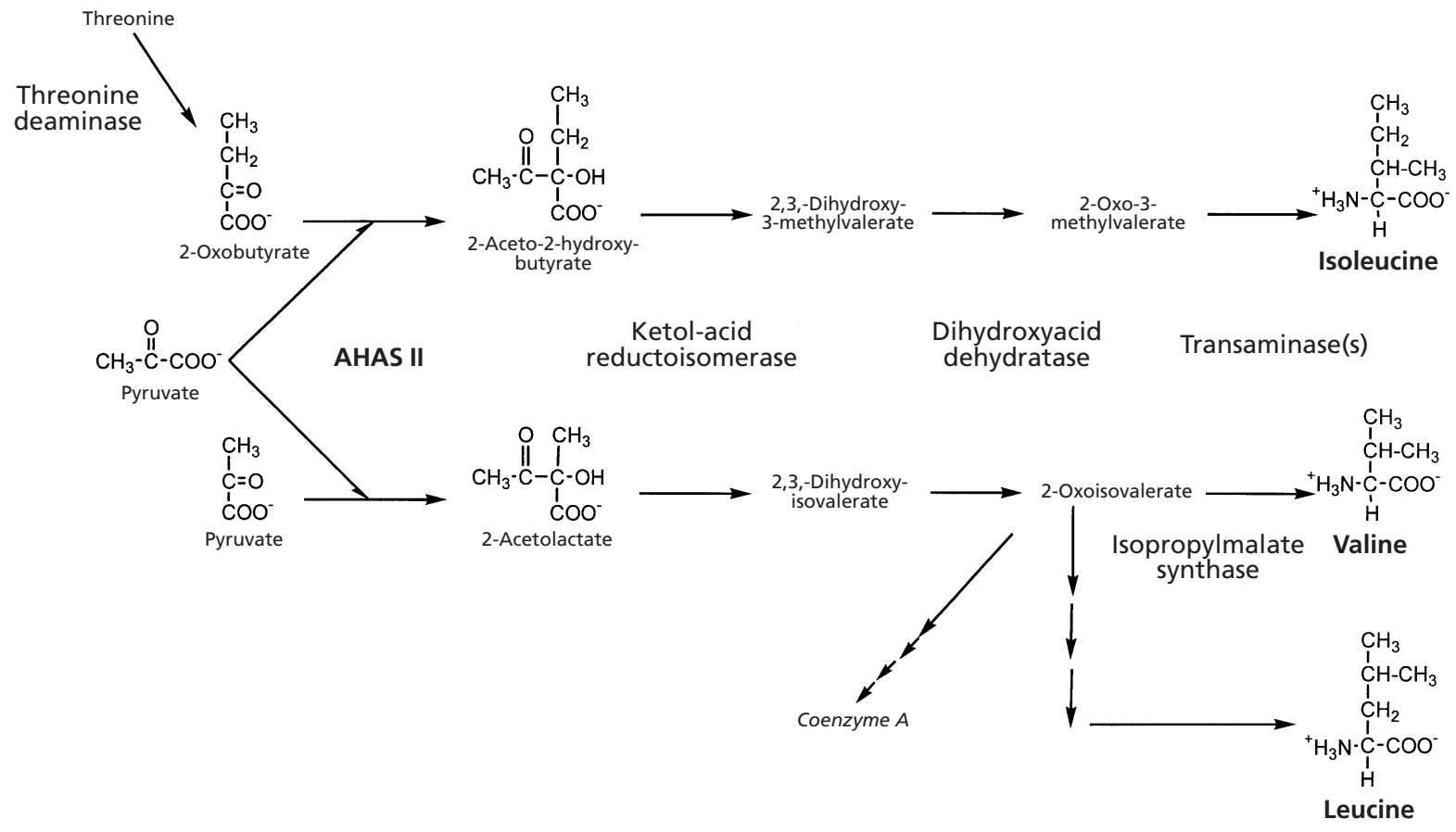

Fig. 1. Biosynthetic pathway of branched-chain amino acids in S. typhimurium TV105.

initiation codon AUG and followed by an out-of-frame lac $Z$ reporter gene. As the reporter gene is in-frame with the expected frameshift, the system allows quantitative determination of translational frameshifting by assay of the reporter gene product, $\beta$-galactosidase. The sequence TTTCAAG led to a -1 translational frameshifting under conditions of starvation for charged tRNA ${ }^{\mathrm{Lys}}$ as a result of ribosome stalling at the lysyl codon, AAG, and frameshifting was correlated directly with the level of starvation (Gallant \& Lindsley, 1992). Changing the codon to the isoleucine-encoding sequences, ATA or ATC, retained the translational frameshifting properties of the system, except that starvation for charged tRNA ${ }^{\text {Ile }}$ was required to initiate frameshifting (Barak et al., 1996). In each case, starvation was achieved by specific inhibition of aminoacyl-tRNA charging by the hydroxamate analogue of the amino acid. In principle, translational frameshifting should also be caused by a deficiency of an amino acid whose codon is in the 'shifty' region, i.e. by inhibition of the biosynthesis of the amino acid.

In this study we demonstrate the use of translational frameshifting as a tool to estimate the degree of starvation for isoleucine in Salmonella typhimurium, when branched-chain amino acid biosynthesis is specifically inhibited by SMM.

\section{METHODS}

Materials. Antibiotics, amino acids, IPTG, ONPG, isoleucine hydroxamate (ILHX) and cAMP were purchased from Sigma.
SMM was a gift of E. I. du Pont de Nemours \& Co., Wilmington, DE, USA. All other chemicals used were of analytical pure grade.

Bacterial strain, growth and media. Salmonella typhimurium LT2-TV105 (LaRossa \& Schloss, 1984) expresses only isozyme II of the three AHAS isozymes of the enteric bacteria (Fig. 1). This isozyme is strongly inhibited by the herbicide SMM and is resistant to valine inhibition (LaRossa \& Schloss, 1984; Schloss et al., 1988).

Plasmid pMLB1115 with the 'shifty' sequence TTTCATA (Barak et al., 1996) was designed for -1 translational frameshifting measurements at the isoleucine codon by means of the $\beta$-galactosidase reporter gene. A strain of $S$. typhimurium TV105 carrying this plasmid was isolated after transformation by electroporation (Pfan \& Youderian, 1990; Takedo, 1988). The transformant was selected to be resistant to kanamycin and ampicillin coded by the host genome and the plasmid, respectively.

S. typhimurium TV105(pMLB1115) was grown in minimal medium M63 (Miller, 1972b) containing kanamycin (50 mg $\left.\mathrm{l}^{-1}\right)$ and ampicillin $\left(150 \mathrm{mg} \mathrm{l}^{-1}\right)$. Cultures were grown with shaking (200 r.p.m.) in a gyratory water bath at $37^{\circ} \mathrm{C}$. Growth was followed by measuring turbidity with a Klett-Summerson colorimeter using a $660 \mathrm{~nm}$ filter.

Determination of translational frameshifting. Translational frameshifting was measured in starved and control cultures by determination of the amount of $\beta$-galactosidase produced after induction with IPTG $(2 \mathrm{mM})$ and cAMP $(2.5 \mathrm{mM})$, in one bacterial generation. The concentrations of the inducers were optimized to give maximal induction without interference with bacterial growth rate (data not shown). $\beta$-Galactosidase activity was measured as described by Miller (1972a). Protein 
concentrations were determined by the Bradford method (Bradford, 1976) using the Bio-Rad Protein Assay Kit with bovine serum albumin as a standard.

\section{RESULTS AND DISCUSSION}

The enterobacteria generally encode three AHAS isozymes, which differ in catalytic properties, regulation and sensitivity to inhibitors (Umbarger, 1987; Barak et al., 1987; Chipman et al., 1998). S. typhimurium strain TV105 is a mutant that expresses AHAS II as the sole isozyme catalysing the first step in the biosynthesis of branched-chain amino acids (LaRossa \& Schloss, 1984). As this isozyme is highly sensitive to the herbicide SMM (LaRossa \& Schloss, 1984; Schloss et al., 1988), the growth of strain TV105 is inhibited by SMM. Cultures continued to grow exponentially after addition of low concentrations of SMM, although at slower rates. As shown in Fig. 2 and previously described (Epelbaum et al., 1996), this inhibition was almost completely relieved by addition of valine alone. Valine itself does not inhibit this bacterium, as AHAS II, unlike most other AHASs, is valine resistant (Schloss \& VanDyk, 1988). This suggests that the inhibition of growth when AHAS activity is decreased is primarily the result of decreased flux in the valine-leucine branch of the branched-chain amino acid path (Fig. 1), and is consistent with the observation that SMM-inhibited S. typhimurium TV105 cells contained reduced amounts of the valine precursor, 2-oxoisovalerate, while the concentration of the parallel isoleucine precursor, 2-oxo-3-methylvalerate, was not reduced (Epelbaum et al., 1996). We suggested (Epelbaum et al., 1996) that the competition between 2-oxobutyrate and pyruvate as AHAS substrates, together with the feedback control of 2-oxobutyrate synthesis by isoleucine, could account for the specific reduction of valine and its precursor, as opposed to isoleucine, when AHAS is inhibited. These findings do not exclude the possibility that accumulation of 2-oxobutyrate could be partly responsible for SMM toxicity through competition with 2-oxoisovalerate, the valine precursor (Fig. 1) (LaRossa et al., 1987; VanDyk \& LaRossa, 1990).

Growth of S. typhimurium TV105(pMLB1115) could be inhibited by SMM even when valine was supplemented, but the concentration of SMM required to achieve a given degree of inhibition was approximately 20 -fold higher in the presence of valine than in its absence (Fig. $3)$. The growth inhibition by SMM observed in the presence of valine is likely to be caused, at least in part, by isoleucine deficiency, as addition of this amino acid to such cultures relieved the toxicity of SMM (Epelbaum et al., 1996, and data not shown). Such deficiency should decrease the level of charged tRNA ${ }^{\text {Ile }}$ and elevate the frequency of translational frameshifting at the isoleucine codon AUA in the 'shifty' region of the mRNA coded by plasmid pMLB1115. The rate of synthesis of $\beta$-galactosidase in S. typhimurium TV105(pMLB1115) indeed increased in cultures inhibited by SMM in the presence of valine (Table 1 ). As expected, the level of translational frameshifting is correlated with the degree of inhibition

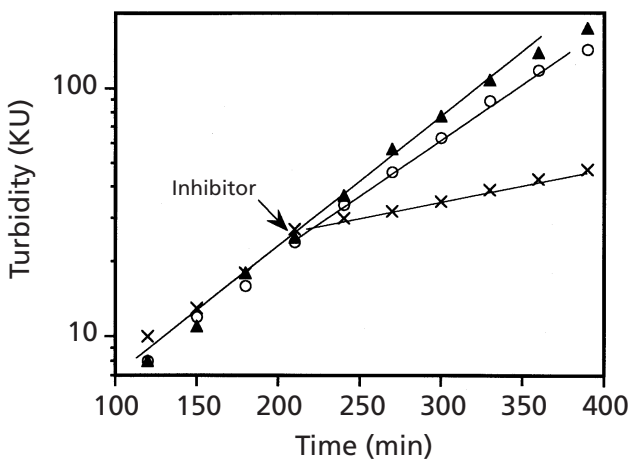

Fig. 2. Influence of valine on SMM inhibition. Growth was followed, as described in Methods, in a control $(\boldsymbol{\Delta})$ culture and cultures to which SMM $(1 \mu \mathrm{M})$ was added alone $(x)$ or simultaneously with $50 \mu \mathrm{g}$ valine $\mathrm{ml}^{-1}(\mathrm{O})$. KU, Klett units.
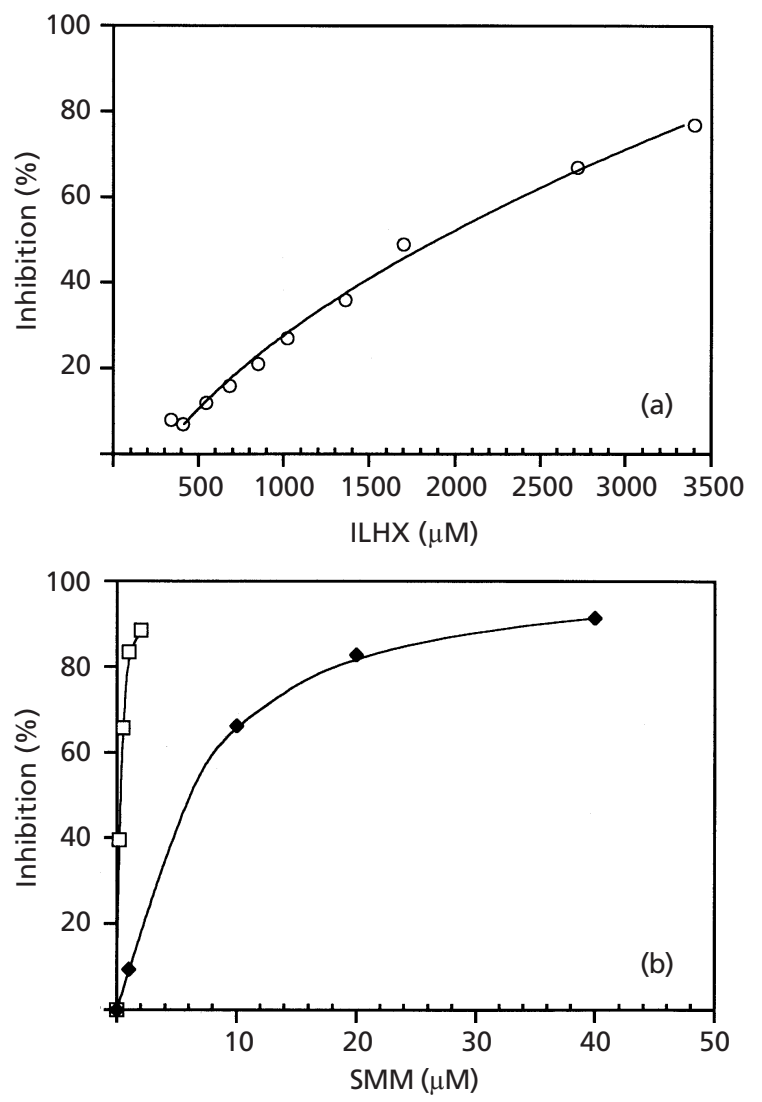

Fig. 3. Inhibition of S. typhimurium TV105(pMLB1115) by ILHX (O) (a) and by SMM with $(\bullet)$ and without $(\square) 50 \mu \mathrm{g}$ valine $\mathrm{ml}^{-1}$ (b). Inhibitors were added to exponentially growing cultures. The degree of inhibition is calculated from the ratio between the growth rates of inhibited and control cultures, grown under conditions as in Fig. 2.

by SMM. The validity of the system in the new host, $S$. typhimurium TV105, was confirmed by the direct correlation obtained between inhibition of growth, 
Table 1. Influence of growth inhibition of S. typhimurium TV105(pMLB1115) on translational frameshifting

Cultures were grown as described in Methods.

\begin{tabular}{|lcccc|}
\hline Inhibitor & Concn $(\boldsymbol{\mu M})$ & Amino acids added $^{*}$ & Inhibition $(\%) \dagger$ & Differential rate $\neq$ \\
\hline None & & - & 0 & 1 \\
ILHX & 680 & - & 16 & $1 \cdot 1 \pm 1 \cdot 0$ \\
& 1700 & - & 49 & $3 \cdot 1 \pm 0 \cdot 8$ \\
SMM & 3400 & - & 77 & $5 \cdot 8 \pm 1 \cdot 0$ \\
& 10 & Val & 66 & $4 \cdot 4 \pm 1 \cdot 8$ \\
& 20 & Val & 83 & $9 \cdot 4 \pm 3 \cdot 0$ \\
& 40 & Val & 92 & $33 \cdot 8 \pm 1 \cdot 7$ \\
& $0 \cdot 5$ & - & 66 & $1 \cdot 3 \pm 0 \cdot 2$ \\
& $1 \cdot 0$ & - & 83 & $0 \cdot 7 \pm 0 \cdot 1$ \\
& 20 & Val, Ile & 0 & $0 \cdot 5 \pm 0 \cdot 1$ \\
\hline
\end{tabular}

*Amino acids were added simultaneously with SMM to final concentrations of $50 \mu \mathrm{g} \mathrm{ml}^{-1}$ each.

† Degree of inhibition was taken from Fig. 3.

$\ddagger$ Translational frameshifting was defined as de novo induced $\beta$-galactosidase synthesis relative to the increase in protein concentration. Values were normalized to the control culture, whose differential rate is taken as 1 .

resulting from charged tRNA ${ }^{\text {Ile }}$ starvation caused by isoleucyl hydroxamate (ILHX), as shown in Fig. 3, and the level of translational frameshifting (Table 1). The levels of ILHX-induced frameshifting in S. typhimurium TV105(pMLB1115) were lower than those observed in Escherichia coli carrying pMLB1115 (Barak et al., 1996). The specificity of this translational frameshifting assay for isoleucine starvation was confirmed by its failure to react at similar degrees of growth inhibition when it was initiated by valine starvation, caused by SMM in minimal medium without valine (Table 1). No elevation in the translational frameshifting level was detected in cultures to which SMM was added in the presence of valine and isoleucine, which protect the bacteria from growth inhibition (Table 1).

In conclusion, we have shown here that a translational frameshifting assay can be adapted for use in bacteria other than E. coli (e.g. S. typhimurium TV105) and used as a measure of starvation for a given amino acid in vivo, induced by metabolic inhibition. We believe this tool can readily be extended to in vivo measurement of starvation for other amino acids caused by various factors, by using constructs with different codons in the 'shifty' region. Translational frameshifting assays could thus be a valuable new tool in the study of metabolic effects of inhibition of enzymes involved in amino acid biosynthesis.

\section{ACKNOWLEDGEMENTS}

This work was partially funded by BGU Seed Grant no. 81824101. We thank J. V. Schloss and DuPont Co. for the SMM, and S. Epelbaum and M. Einav for their assistance.

\section{REFERENCES}

Atkins, J. F., Weiss, R. B. \& Gesteland, R. F. (1990). Ribosome gymnastics. Cell 62, 413-423.

Barak, Z., Chipman, D. M. \& Gollop, N. (1987). Physiological implications of the specificity of acetohydroxy acid synthase isozymes of enteric bacteria. J Bacteriol 169, 3750-3756.

Barak, Z., Gallant, J. A. \& Lindsley, D. (1996). Leftward frameshifting at several codons. J Mol Biol 256, 676-684.

Bradford, M. M. (1976). A rapid and sensitive method for the quantitation of microgram quantities of protein utilizing the principle of protein-dye binding. Anal Biochem 72, 248-254.

Chaleff, R. S. \& Mauvais, C. J. (1984). Acetolactate synthase is the site of action of two sulfonylurea herbicides in higher plants. Science 224, 1443-1445.

Chipman, D., Barak, Z. \& Schloss, J. V. (1998). Biosynthesis of 2aceto-2-hydroxy acids: acetolactate synthases and acetohydroxyacid synthases. Biochim Biophys Acta 1385, 401-419.

Epelbaum, S., Chipman, D. M. \& Barak, Z. (1996). Metabolic effects of inhibitors of two enzymes of the branched-chain amino acid pathway in Salmonella typhimurium. J Bacteriol 178, 1187-1196.

Epelbaum, S., LaRossa, R. A., VanDyk, T., Elkayam, T., Chipman, D. M. \& Barak, Z. (1998). Branched-chain amino acid biosynthesis in Salmonella typhimurium: a quantitative analysis. J Bacteriol 180, 4056-4067.

Gallant, J. A. \& Lindsley, D. (1992). Leftward ribosome frameshifting at a hungry codon. $J$ Mol Biol 223, 31-40.

Ivanov, I. P., Gesteland, R. F. \& Atkins, J. F. (2000). Antizyme expression: a subversion of triplet decoding, which is remarkably conserved by evolution, is a sensor for an autoregulatory circuit. Nucleic Acids Res 28, 3185-3196.

Jia, M. H., LaRossa, R. A., Lee, J., Rafalski, A., DeRose, E., Gonye, G. \& Xue, Z. (2000). Global expression profiling of yeast treated 
with an inhibitor of amino acid biosynthesis, sulfometuron methyl. Physiol Genomics 3, 83-92.

LaRossa, R. A. \& Schloss, J. V. (1984). The sulfonylurea herbicide sulfometuron methyl is an extremely potent and selective inhibitor of acetolactate synthase in Salmonella typhimurium. J Biol Chem 259, 8753-8757.

LaRossa, R. A., VanDyk, T. K. \& Smulski, D. R. (1987). Toxic accumulation of $\alpha$-ketobutyrate caused by inhibition of the branched-chain amino acid biosynthetic enzyme acetolactate synthase in Salmonella typhimurium. J Bacteriol 169, 1372-1378.

Miller, J. H. (1972a). In Experiments in Molecular Genetics, pp. 352-356. Cold Spring Harbor, NY: Cold Spring Harbor Laboratory.

Miller, J. H. (1972b). In Experiments in Molecular Genetics, p. 431. Cold Spring Harbor, NY: Cold Spring Harbor Laboratory.

Parker, G. (1989). Errors and alternatives in reading the universal genetic code. Microbiol Rev 53, 273-298.

Pfan, J. \& Youderian, P. (1990). Transferring plasmid DNA between different bacterial species with electroporation. Nucleic Acids Res 18, 6165.

Schloss, J. V. (1984). Interaction of the herbicide sulfometuron methyl with acetolactate synthase: a slow-binding inhibitor. In
Flavins and Flavoproteins, pp. 737-740. Edited by R. C. Bray, P. C. Engel \& S. G. Mayhew. Berlin: de Gruyter.

Schloss, J. V. \& VanDyk, D. E. (1988). Acetolactate synthase isozyme II from Salmonella typhimurium. Methods Enzymol 166, 445-454.

Schloss, J. V., Ciskanik, L. M. \& VanDyk, D. E. (1988). Origin of the herbicide binding site of acetolactate synthase. Nature 331, 360-362.

Takedo, A. (1988). DNA transfection of E. coli by electroporation. Biochim Biophys Acta 949, 318-324.

Umbarger, H. E. (1987). Biosynthesis of the branched chain amino acids. In Escherichia coli and Salmonella typhimurium: Cellular and Molecular Biology, pp. 352-367. Edited by F. C. Neidhardt and others. Washington, DC: American Society for Microbiology.

VanDyk, T. K. \& LaRossa, R. A. (1990). Prevention of endogenous 2-ketobutyrate toxicity in Salmonella typhimurium. In Biosynthesis of Branched Chain Amino Acids, pp. 123-130. Edited by Z. Barak, D. M. Chipman \& J. V. Schloss. Weinheim: VCH.

Received 5 July 2001; revised 29 October 2001; accepted 16 November 2001. 